\title{
Um método do ponto proximal inexato para problemas de quase-equilíbrio
}

Edimilson Lopes Dias Júnior ${ }^{1}$

DM/UFPI, Teresina, PI

Pedro Jorge S. Santos ${ }^{2}$

UFDPar, Parnaíba, PI

João Carlos de Oliveira Souza ${ }^{3}$

DM/UFPI, Teresina, PI

Resumo. Neste trabalho, propomos uma versão inexata do método do ponto proximal para problemas de quase-equilíbrio (QEP) considerado em Santos e Souza [A proximal point method for quasi-equilibrium problems in Hilbert spaces, Optimization, 1-16, 2020]. Analisamos as propriedades do método proposto e provamos, sob hipóteses razoáveis, sua convergência para uma solução do problema. Por fim, um experimento numérico ilustra os resultados obtidos.

Palavras-chave. Problema de quase-equilíbrio, método do ponto proximal, problema de equilíbrio.

\section{Introdução}

Neste trabalho, estudaremos um método inexato para resolver problemas de quase-equilíbrio (QEP). Para isso, consideramos $X \subset \mathbb{R}^{n}$ um conjunto convexo, fechado e não-vazio. Denotaremos por $f: X \times X \rightarrow \mathbb{R}$ uma bifunção de equilíbrio, ou seja, uma função que satisfaz $f(x, x)=0$ para todo $x \in X$. Antes de definir o que será um problema de quase-equilíbrio, introduziremos alguns conceitos relacionados a problemas de equilíbrio (EP). Tais conceitos servirão de suporte para as definições e resultados que seguem. O problema de equilíbrio, denotado por $E P(f, X)$, consiste de encontrar um ponto $x^{*} \in X$ tal que

$$
f\left(x^{*}, y\right) \geq 0, \quad \forall y \in X
$$

O conjunto solução de $E P(f, X)$ será denotado por $S_{E P}(f, X)$. Ao $E P(f, X)$ podemos associar um novo problema, que consiste em encontrar $y^{*} \in X$ satisfazendo

$$
f\left(x, y^{*}\right) \leq 0, \quad \forall x \in X
$$

Este problema é conhecido como o problema dual de $E P(f, X)$. Analogamente, seu conjunto solução será denotado por $S_{E P}^{d}(f, X)$. Os problemas de equilíbrio tem uma vasta aplicação em matemática, podendo ser utilizados para modelar as mais diversas situações, tais como problemas de otimização, desigualdades variacionais, complementaridade, otimização vetorial e teoria dos jogos não cooperativos. Veja por exemplo Blum e Oettli [1].

\footnotetext{
1 edimilson.lopes@ufpi.edu.br

2 pedrojorge@ufpi.edu.br

3 joaocos.mat@ufpi.edu.br
} 
Uma vez definido o problema de equilíbrio, passemos a um problema mais geral chamado de problema de quase-equilíbrio. Um problema de quase-equilíbrio, o qual denotaremos por $Q E P(f, C)$, consiste de encontrar um ponto $x^{*} \in C\left(x^{*}\right)$ tal que

$$
f\left(x^{*}, y\right) \geq 0, \quad \forall y \in C\left(x^{*}\right)
$$

onde $C: X \rightrightarrows X$ é uma aplicação ponto-conjunto que associa a cada ponto de $X$ um subconjunto em $X$. Observe que, quando $C(x) \equiv X$, para todo $x \in X$, o problema de quase-equilíbrio retorna ao problema de equilíbrio (1). Denotaremos o conjunto solução do problema de quase-equilíbrio por $S_{Q E P}(f, C)$. É bem conhecido que problemas tais como desigualdades quase variacionais, jogos de Nash generalizados e outros, não se encaixam na formatação de (1) e sim na formatação de quaseequilíbrio (2). Daí surge a necessidade de uma generalização do então problema de equilíbrio para o quase-equilíbrio. Veja, por exemplo, Santos e Souza [5] e suas referências.

Uma das técnicas mais empregadas para resolver um problema de equilíbrio é o método do ponto proximal que através de uma regularização resolve em cada iteração um novo problema de equilíbrio, chamado problema regularizado e denotado por $E P\left(f_{k}, X\right)$, onde a nova bifunção $f_{k}$ nada mais é que a bifunção do problema original acrescida da regularização. Em nosso trabalho, propomos uma versão inexata do método do ponto proximal para problemas de quase-equilíbrio desenvolvido por Santos e Souza [5], no sentido de que a função regularização $f_{k}^{e}$ dada por

$$
f_{k}^{e}(x, y)=f(x, y)+\gamma_{k}\left\langle x-x^{k}, y-x\right\rangle-\left\langle e^{k}, y-x\right\rangle
$$

$\operatorname{com}\left\{\gamma_{k}\right\} \subset \mathbb{R}$ uma sequência positiva e limitada, de certa forma, generaliza a regularização considerada em [5], uma vez que fazendo $e^{k}=0$, para todo $k \in \mathbb{N}$, a bifunção regularizada considerada neste trabalho coincide com a proposta em [5]. Caso contrário, os subproblemas resolvidos pelo nosso método são inexatos o que dará maior flexibilidade ao método, principalmente do ponto de vista computacional.

Nosso trabalho se apresenta dividido da seguinte maneira: Na Seção 2 apresentaremos resultados preliminares relativos a EP e QEP. Na Seção 3 apresentaremos o algoritmo proposto e analisaremos suas propriedades de convergência. Por fim, na Seção 4 realizamos um experimento numérico para verificar a eficiência do método proposto. Finalizamos com algumas considerações finais na Seção 5.

\section{Preliminares}

Seja $X \subset \mathbb{R}^{n}$ um conjunto não vazio, convexo e fechado. No decorrer desse trabalho assumiremos que a aplicação $f: X \times X \rightarrow \mathbb{R}$ satisfaz as seguintes hipóteses as quais são amplamente adotadas na literatura de problemas de equilíbrio e quase-equilíbrio.

(P1) $f(x, x)=0$, para todo $x \in X$;

(P2) $f(\cdot, \cdot): X \times X \rightarrow \mathbb{R}$ é contínua em $X \times X$;

(P3) $f(x, \cdot): X \rightarrow \mathbb{R}$ é convexa, para todo $x \in X$;

(P4) $f$ é monótona, i.e., $f(x, y)+f(y, x) \leq 0$ para quaisquer $x, y \in X$;

Proposição 2.1. Considere $f: X \times X \rightarrow \mathbb{R}$ satisfazendo (P1)-(P4). Fixe $\bar{x}$, e $\in \mathbb{R}^{n}$ e $\gamma>0$. Seja $f^{e}: X \times X \rightarrow \mathbb{R}$ dada por

$$
f^{e}(x, y)=f(x, y)+\gamma\langle x-\bar{x}, y-x\rangle-\langle e, y-x\rangle,
$$

então $\operatorname{EP}\left(f^{e}, X\right)$ tem solução única. 
Demonstração. Veja [2, Proposição 3.1].

A seguir apresentamos a definição de M-continuidade (ou continuidade no sentido de Mosco); veja Mosco [3]. Esse conceito será usado nos resultados referentes à análise de convergência do método.

Definição 2.1. Dizemos que uma aplicação $C: X \rightrightarrows X$ é $M$-contínua se:

(i) Dados $\left\{x^{k}\right\},\left\{y^{k}\right\} \subset X$, com $y^{k} \in C\left(x^{k}\right), x^{k} \rightarrow x$ e $y^{k} \rightarrow y$, então $y \in C(x)$;

(ii) Para qualquer sequência $\left\{x^{k}\right\} \subset X$, tal que $x^{k} \rightarrow x$, e para todo $y \in C(x)$, existe uma sequência $\left\{y^{k}\right\} \subset X$ tal que $y^{k} \in C\left(x^{k}\right)$ e $y^{k} \rightarrow y$.

\section{Método do ponto proximal inexato}

Nesta seção, definimos uma versão inexata do método ponto proximal (MPP) para problemas de quase-equilíbrio proposto por Santos e Souza [5] propiciando ao método maior aplicabilidade e flexibilidade, principalmente do ponto de vista computacional. Dessa forma, iremos supor que $f$ satisfaz (P1)-(P4) e que $C$ é M-contínua.

\section{MPP inexato}

Passo 1: Tome uma sequência limitada de escalares positivos $\left\{\gamma_{k}\right\}_{k \in \mathbb{N}}$ e escolha $x^{0} \in X$;

Passo 2: Dado $x^{k}$, calcule $x^{k+1} \in S_{E P}\left(f_{k}^{e^{k}}, C\left(x^{k}\right)\right)$, onde

$$
f_{k}^{e^{k}}(x, y)=f(x, y)+\gamma_{k}\left\langle x-x^{k}, y-x\right\rangle-\left\langle e^{k}, y-x\right\rangle
$$

com

$$
\left\|e^{k}\right\| \leq\left\|x^{k+1}-x^{k}\right\|
$$

Passo 3: Se $x^{k+1}=x^{k}$, pare e retorne $x^{k}$. Caso contrário, faça $k=k+1$ e retorne ao Passo 2.

Observação 3.1. Note que em cada subproblema do método resolvemos um problema de equilíbrio visto que o conjunto $C_{k}:=C\left(x^{k}\right)$ está fixo, para cada $k \in \mathbb{N}$. Dessa forma, sob as hipóteses (P1)-(P4), o problema de encontrar $x^{k+1} \in S_{E P}\left(f_{k}^{e^{k}}, C_{k}\right)$ tem solução única e com isso o método está bem definido; veja Proposição 2.1.

Uma vez bem definido o algoritmo, estudaremos algumas de suas propriedades de convergência. Para isso, iniciamos com o seguinte resultado que diz respeito ao critério de parada do método.

Proposição 3.1. Se $x^{k+1}=x^{k}$ para algum $k \in \mathbb{N}$, então $x^{k}$ é solução do QEP.

Demonstração. Pela definição do algoritmo, temos que $x^{k+1} \in S_{E P}\left(f_{k}^{e^{k}}, C_{k}\right)$. Assim, temos que $x^{k+1} \in C_{k}$ e $f_{k}^{e^{k}}\left(x^{k+1}, y\right) \geq 0$, para todo $y \in C_{k}$. Portanto,

$$
f\left(x^{k+1}, y\right)+\gamma_{k}\left\langle x^{k+1}-x^{k}, y-x^{k+1}\right\rangle-\left\langle e^{k}, y-x^{k+1}\right\rangle \geq 0, \quad \forall y \in C_{k} .
$$

Sendo $x^{k+1}=x^{k}$, segue de (4) que $e^{k}=0$ e, além disso, $\gamma_{k}\left\langle x^{k+1}-x^{k}, y-x^{k+1}\right\rangle=0$. Assim, obtemos de (5) que $f\left(x^{k+1}, y\right) \geq 0$, para todo $y \in C\left(x^{k}\right)$. Logo, obtemos que $x^{k} \in C\left(x^{k}\right)$ e $f\left(x^{k}, y\right) \geq 0$ para todo $y \in C\left(x^{k}\right)$. Portanto, $x^{k} \in S_{Q E P}(f, C)$ e a prova da proposição está completa. 
Em virtude da proposição anterior, iremos supor que o algoritmo gera uma sequência $\left\{x^{k}\right\}$ infinita. Uma propriedade clássica e desejável, se tratando de método do ponto proximal, é conhecida como convergência assintótica. Neste trabalho, iremos supor que a sequência $\left\{x^{k}\right\}$ gerada pelo Algoritmo MPP Inexato satisfaz tal propriedade, definida abaixo, que iremos chamar de (H1).

(H1) Seja $\left\{x^{k}\right\}$ a sequência gerada pelo algoritmo, então

$$
\lim _{k \rightarrow+\infty}\left\|x^{k+1}-x^{k}\right\|=0 .
$$

Teorema 3.1. Todo ponto de acumulação de $\left\{x^{k}\right\}$ pertence a $S_{Q E P}(f, C)$

Demonstração. Seja $\widehat{x}$ um ponto de acumulação qualquer de $\left\{x^{k}\right\}$ e considere $\left\{x^{k_{j}}\right\}$ uma subsequência de $\left\{x^{k}\right\}$ que converge para $\widehat{x}$. Da definição do algoritmo, temos que $x^{k_{j+1}} \in C\left(x^{k_{j}}\right)$. De (H1) temos que $x^{k_{j+1}} \rightarrow \widehat{x}$. Logo, da M-Continuidade de $C$, temos que $\widehat{x} \in C(\widehat{x})$. Novamente pela M-continuidade de $C$, temos que, dado $y \in C(\widehat{x})$ arbitrário, existe uma sequência $\left\{y^{k_{j}}\right\}$ tal que $y^{k_{j}} \rightarrow y$ e $y^{k_{j}} \in C\left(x^{k_{j}}\right)$. Agora, como $x^{k_{j+1}} \in S_{E P}\left(f_{k_{j}}^{e^{k_{j}}}, C_{k_{j}}\right)$ temos

$$
f_{k_{j}}^{e^{k_{j}}}\left(x^{k_{j+1}}, z\right) \geq 0, \quad \forall z \in C\left(x^{k_{j}}\right) .
$$

$\mathrm{O}$ que significa que

$$
f\left(x^{k_{j+1}}, z\right)+\gamma_{k_{j}}\left\langle x^{k_{j+1}}-x^{k_{j}}, z-x^{k_{j+1}}\right\rangle-\left\langle e^{k_{j}}, z-x^{k_{j+1}}\right\rangle \geq 0, \quad \forall z \in C\left(x^{k_{j}}\right) .
$$

Em particular, para $z=y^{k_{j}}$, temos

$$
\begin{aligned}
0 & \leq f\left(x^{k_{j+1}}, y^{k_{j}}\right)+\gamma_{k_{j}}\left\langle x^{k_{j+1}}-x^{k_{j}}, y^{k_{j}}-x^{k_{j+1}}\right\rangle-\left\langle e^{k_{j}}, y^{k_{j}}-x^{k_{j+1}}\right\rangle \\
& \leq f\left(x^{k_{j+1}}, y^{k_{j}}\right)+\gamma_{k_{j}}\left\|x^{k_{j+1}}-x^{k_{j}}\right\|\left\|y^{k_{j}}-x^{k_{j+1}}\right\|+\left\|e^{k_{j}}\right\|\left\|x^{k_{j+1}}-y^{k_{j}}\right\| .
\end{aligned}
$$

Usando o fato de que $\left\{\gamma_{k_{j}}\right\},\left\{y^{k_{j}}\right\}$ e $\left\{x^{k_{j}}\right\}$ são limitadas e que (H1) fornece $\lim _{j \rightarrow+\infty}\left\|x^{k_{j+1}}-x^{k_{j}}\right\|=0$, e consequentemente de (4) segue que $\lim _{j \rightarrow+\infty}\left\|e^{k_{j}}\right\|=0$. Tomando o limite com $j \rightarrow+\infty$ em (6) e levando em conta que $\left\{\left\|y^{k_{j}}-x^{k_{j+1}}\right\|\right\}$ é limitada, segue de (P2) que

$$
f(\widehat{x}, y) \geq 0, \quad \forall y \in C(\widehat{x}),
$$

obtendo que $\widehat{x} \in S_{Q E P}(f, C)$.

O resultado seguinte fornece uma caracterização para que um ponto $x^{*}$ seja solução de um problema de quase-equilíbrio. Usaremos esse resultado para obter, nos experimentos numéricos, uma precisão do ponto limite obtido pelo método. Esse resultado foi provado em Santos e Souza [5] e, a seguir, apresentamos sua prova por questão de completeza do trabalho.

Seja $f: X \times X \rightarrow \mathbb{R}$ uma bifunção. Denotaremos por $\partial_{2} f$ o subdiferencial (no sentido clássico de análise convexa) de $f$ com respeito a segunda variável. Uma vez que valem (P1) e (P3) temos

$$
\begin{aligned}
\partial_{2} f(x, x) & =\{v \in H: f(x, y) \geq f(x, x)+\langle v, y-x\rangle\} \\
& =\{v \in H: f(x, y) \geq\langle v, y-x\rangle\} .
\end{aligned}
$$

Dado $x \in X$, denotamos por $\Upsilon(x)=\left\|x-\arg \min _{y \in C(x)}\left\{f(x, y)+\frac{1}{2}\|y-x\|^{2}\right\}\right\|$.

Proposição 3.2. Um ponto $x^{*} \in S_{Q E P}(f, C)$ se, somente se, $\Upsilon\left(x^{*}\right)=0$ 
Demonstração. Suponha que $x^{*} \in S_{Q E P}(f, C)$. Então, $x^{*} \in C\left(x^{*}\right)$ e

$$
f\left(x^{*}, y\right)+\frac{1}{2}\left\|y-x^{*}\right\|^{2} \geq f\left(x^{*}, y\right) \geq 0 .
$$

Agora note que $0=f\left(x^{*}, x^{*}\right)+\frac{1}{2}\left\|x^{*}-x^{*}\right\|^{2}$, isso nos diz que

$$
x^{*}=\arg \min _{y \in C\left(x^{*}\right)}\left\{f\left(x^{*}, y\right)+\frac{1}{2}\left\|y-x^{*}\right\|^{2}\right\}
$$

e com isso, obtemos que $\Upsilon\left(x^{*}\right)=0$.

Agora, se $x^{*}=\arg \min _{y \in C\left(x^{*}\right)}\left\{f\left(x^{*}, y\right)+\frac{1}{2}\left\|y-x^{*}\right\|^{2}\right\}$, temos, da condição de otimalidade de primeira ordem, que $0 \in \partial_{2} f\left(x^{*}, x^{*}\right)+N_{C\left(x^{*}\right)}\left(x^{*}\right)$. Assim, temos que existe $s^{*} \in \partial_{2} f\left(x^{*}, x^{*}\right)$ tal que $0 \in s^{*}+N_{C\left(x^{*}\right)}\left(x^{*}\right)$. O que por sua vez implica que $-s^{*} \in N_{C\left(x^{*}\right)}\left(x^{*}\right)$. Da definição de cone normal, temos $\left\langle y-x^{*},-s^{*}\right\rangle \leq 0, \quad \forall y \in C\left(x^{*}\right)$. Consequentemente, obtemos que

$$
\left\langle y-x^{*}, s^{*}\right\rangle \geq 0, \quad \forall y \in C\left(x^{*}\right) .
$$

Por outro lado, $s^{*} \in \partial_{2} f\left(x^{*}, x^{*}\right)$ e, de (P3), temos que $f\left(x^{*}, \cdot\right)$ é convexa, logo

$$
f\left(x^{*}, y\right) \geq f\left(x^{*}, x^{*}\right)+\left\langle s^{*}, y-x^{*}\right\rangle=\left\langle s^{*}, y-x^{*}\right\rangle \geq 0, \quad \forall y \in C\left(x^{*}\right),
$$

onde a última desigualdade segue usando, de (P1), o fato que $f\left(x^{*}, x^{*}\right)=0$ e (7). Portanto, temos que $x^{*} \in S_{Q E P}(f, C)$.

\section{Ilustração Numérica}

Nesta seção ilustramos numericamente o método proposto através de uma versão QEP de um problema originalmente apresentado como EP em [4, Exemplo 4] e adaptado para QEP por Santos e Souza [5, Exemplo 4.1]. Uma vez que o método proposto não requer diferenciabilidade para o problema, o exemplo aqui apresentado foi escolhido por ser um QEP com dados não suaves.

O algoritmo foi escrito no MATLAB ${ }^{\circledR}$ R2017b em um computador com 8 GB RAM e Intel Core i7. O critério de parada adotado foi $\left\|x^{k+1}-x^{k}\right\|<t_{\text {qep }}$ e erro não superior a $\frac{1}{2}\left\|x^{k+1}-x^{k}\right\|$. Utilizamos $\gamma_{k}=1$, para todo $k \in \mathbb{N}$, e resolvemos o subproblema do Passo 2 por meio de um método de subgradiente projetado (IPSM) introduzido por [4], o qual permite tratar com bifunções de equilíbrio possivelmente não diferenciáveis. Com respeito ao algoritmo IPSM, adotamos $\epsilon_{k}=$ $\xi_{k}=0, \rho_{k}=3$ e $\beta_{k}=\frac{10}{3 k}$. A projeção requerida pelo IPSM foi resolvida utilizando a subrotina "quadprog" do MATLAB e o critério de parada utilizado foi o mesmo de [4] com $\varepsilon=t_{\text {e }}$.

Exemplo 4.1. [4, Exemplo 4-versão QEP] Considere o problema de quase-equilíbrio não suave de dimensão 2 definido pela bifunção

$$
f(x, y)=\left|y_{1}\right|-\left|x_{1}\right|+y_{2}^{2}-x_{2}^{2}
$$

e a aplicação ponto-conjunto $C$ dada por

$$
C(x)=\left\{y \in \mathbb{R}_{+}^{2} ; y_{1}+y_{2}=1+\frac{\left|x_{1}\right|}{1+\left|x_{1}\right|}\right\} .
$$

Observe que a única solução do problema é o ponto $x^{*}=\left(1, \frac{1}{2}\right)$. 
Na Tabela 1, apresentamos cada iterada $x^{k}$, a precisão $\Upsilon\left(x^{k}\right)$ (veja Proposição 3.2) e a norma $\left\|x^{k+1}-x^{k}\right\|$ (veja hipótese H1) obtida pelo algoritmo considerando como ponto inicial $x^{0}=(0,0)$. Na Tabela 2, apresentamos o resultado médio obtido pelo algoritmo ao utilizar 100 pontos iniciais aleatórios tomados na caixa $[-5,5]^{2}$. As colunas informam o mínimo, o máximo e a média das iterações além do tempo de CPU até o critério de parada ser satisfeito. Além disso, apresentamos a média tanto da precisão $\Upsilon\left(x^{k}\right)$ bem como da norma $\left\|x^{k+1}-x^{k}\right\|$ avaliados na última iterada $x^{k}$.

Tabela 1: PPM-inexato com tol $_{q e p}=\operatorname{tol}_{e p}=10^{-6}$ e $x^{0}=(0,0)$.

\begin{tabular}{|c|c|c|c|c|}
\hline Iter.(k) & $x_{1}^{k}$ & $x_{2}^{k}$ & $\left\|x^{k+1}-x^{k}\right\|$ & $\Upsilon\left(x^{k}\right)$ \\
\hline 0 & 0 & 0 & 0.70710678 & - \\
\hline 1 & 0.50000000 & 0.50000000 & 0.33310648 & 0.33333333 \\
\hline 2 & 0.83310569 & 0.50022764 & 0.12123194 & 0.12137224 \\
\hline 3 & 0.95433690 & 0.50014081 & 0.03390424 & 0.03398088 \\
\hline 4 & 0.98824038 & 0.50007711 & 0.00876260 & 0.00880266 \\
\hline 5 & 0.99700220 & 0.50004051 & 0.00222701 & 0.00224759 \\
\hline 6 & 0.99922844 & 0.50002099 & 0.00056833 & 0.00057898 \\
\hline 7 & 0.99979598 & 0.50001106 & 0.00014809 & 0.00015341 \\
\hline 8 & 0.99994332 & 0.50000567 & 0.00004038 & 0.00004289 \\
\hline 9 & 0.99998303 & 0.50000280 & 0.00001193 & 0.00001303 \\
\hline 10 & 0.99999446 & 0.50000130 & 0.00000391 & 0.00000436 \\
\hline 11 & 0.99999804 & 0.50000057 & 0.00000136 & 0.00000157 \\
\hline 12 & 0.99999913 & 0.50000039 & 0.00000063 & 0.00000076 \\
\hline
\end{tabular}

Tabela 2: Resultados obtidos utilizando 100 pontos iniciais aleatórios tomados na caixa $[-5,5]^{2}$, tol $_{e p}=10^{-4}$ e tol $_{q e p}=10^{-6}$.

\begin{tabular}{cccc}
\hline min iter.(k) & max iter.(k) & med. iter. $(\mathrm{k})$ & $\|$ med. $\left\|x^{k}-x^{k-1}\right\|$ \\
\hline 10 & 14 & 10.53 & $5.1926 \mathrm{e}-07$ \\
\hline \hline $\min \mathrm{cpu}(\mathrm{s})$ & $\max \mathrm{cpu}(\mathrm{s})$ & med. $\mathrm{cpu}(\mathrm{s})$ & $\operatorname{med} \Upsilon \Upsilon\left(x^{k}\right)$ \\
\hline 0.6594 & 2.3672 & 1.6803 & $6.7740 \mathrm{e}-07$ \\
\hline
\end{tabular}

\section{Conclusões}

Neste trabalho, apresentamos uma versão inexata de um método do ponto proximal para resolver problemas de quase-equilíbrio. Numericamente, no exemplo implementado, o método obteve performance similar à sua versão exata, tanto em número de iteradas quanto em tempo de CPU, mesmo calculando os subproblemas de forma aproximada. Os trabalhos futuros irão considerar condições suficientes para que a hipótese (H1) se verifique, além de outras regularizações e mais teste numéricos para comprovar a eficácia do método. 


\section{Agradecimentos}

E.L. Dias Júnior foi parcialmente financiado pela Coordenação de Aperfeiçoamento de Pessoal de Nível Superior (CAPES). J.C.O. Souza foi financiado em partes por CNPq $n^{\circ}$ 313901/2020-1.

\section{Referências}

[1] Blum, E. and Oettli, W. From optimization and variational inequalities to equilibrium problems, The Mathematics Student, 63(1-4):123-145, 1994.

[2] Iusem, A.N. and Nasri, M. Inexact proximal point methods for equilibrium problems in Banach spaces, Numer. Funct. Anal. Optim., 28:1279-1308, 2007. DOI:10.1080/01630560701766668

[3] Mosco, U. Convergence of convex sets and of solutions of variational inequalities, Adv. Math., 3:512-585, 1969. DOI:10.1016/0001-8708(69)90009-7

[4] Santos, P.S.M. and Scheimberg, S. An inexact subgradient algorithm for equilibrium problems, J. Comput. Appl. Math., 30:91-107, 2011. DOI:10.1590/S1807-03022011000100005

[5] Santos, P.J.S. and Souza, J.C.O. A proximal point method for quasi-equilibrium problems in Hilbert spaces, Optimization, 1-16, 2020. DOI: 10.1080/02331934.2020.1810686 